\title{
Sperm arylsulfatase A binds to mZP2 and mZP3 glycoproteins in a nonenzymatic manner
}

\author{
Hongbin $\mathrm{Xu}^{1,2}$, Fang Liu ${ }^{1,2}$, Nopparat Srakaew ${ }^{1}$, Chaitanya Koppisetty ${ }^{4,5}$, Per-Georg Nyholm ${ }^{4,6}$, \\ Euridice Carmona ${ }^{7,8}$ and Nongnuj Tanphaichitr ${ }^{1,2,3}$ \\ ${ }^{1}$ Chronic Disease Program, Ottawa Hospital Research Institute, Ottawa, Ontario, Canada, Departments of \\ ${ }^{2}$ Biochemistry/Microbiology/Immunology and ${ }^{3}$ Obstetrics/Gynecology, Faculty of Medicine, University of Ottawa, \\ Ottawa, Ontario, Canada, ${ }^{4}$ Biognos AB, Gothenburg, Sweden, ${ }^{5}$ Department of Computing Science, Chalmers \\ University of Technology, Gothenburg, Sweden, ${ }^{6}$ Institute of Biomedicine, University of Gothenburg, Gothenburg, \\ Sweden, ${ }^{7}$ Maisonneuve-Rosemont Hospital Research Center, Montreal, Quebec, Canada and ${ }^{8}$ Department of \\ Medicine, University of Montreal, Montreal, Quebec, Canada
}

Correspondence should be addressed to N Tanphaichitr at Ottawa Hospital Research Institute; Email: ntanphaichitr@ohri.ca

\begin{abstract}
We have shown previously that sperm surface arylsulfatase A (ASA) of mouse, pig, and human is involved in sperm-egg zona pellucida (ZP) binding. By treating capacitated mouse sperm with A23187 to induce the acrosome reaction, we demonstrated by immunoblotting that ASA also existed in the acrosomal content and on the inner acrosomal membrane. Since mZP2 and mZP3 are known as sperm receptors, whereas $\mathrm{mZP1}$ as a cross-linker of $\mathrm{mZP2} / \mathrm{mZP3}$, we determined whether purified ASA bound to $\mathrm{mZP2}$ and $\mathrm{mZP3}$ selectively. The three mZP glycoproteins were purified from solubilized ovarian ZP by size exclusion column chromatography. Immuno-dot blot analyses revealed that purified sperm ASA bound to mZP2 at the highest level followed by mZP3, whereas the binding of ASA to mZP1 was minimal. The results confirmed the physiological significance of sperm ASA in the ZP binding process. The binding of ASA to mZP2 and mZP3 was, however, not dependent on the active site pocket amino acids, Cys69, Lys123, and Lys302, which are pertinent to the capturing of an arylsulfate substrate, since ASA mutant with Ala substitution at these three residues still bound to mZP2 and $\mathrm{mZP3}$. The availability of the active site pocket of ASA bound to the ZP suggested that ASA would still retain enzymatic activity, which might be important for subsequent sperm penetration through the ZP.

Reproduction (2012) $\mathbf{1 4 4} 209-219$
\end{abstract}

\section{Introduction}

Gamete interaction that culminates in fertilization starts with specific interaction between sperm and the zona pellucida (ZP). Consistently, the ZP of all mammals consists of three to four sulfoglycoproteins; these are mZP1 ( $200 \mathrm{kDa}$ - dimeric form), mZP2 (120 kDa), and mZP3 (83 kDa) in mice. mZP2 and mZP3 form fibrils that are cross-linked by mZP1 (Wassarman \& Litscher 2008, Litscher et al. 2009). Early work of Wassarman and colleagues indicate that mZP3 is the primary receptor of acrosome intact $(\mathrm{Al})$ sperm, whereas $\mathrm{mZP} 2$ is the secondary receptor for acrosome reacted (AR) sperm, and that the glycans of both mZP3 and mZP2 are essential for sperm binding (Wassarman \& Litscher 2008, Litscher et al. 2009). mZP3 also induces the acrosome reaction of capacitated sperm (Florman \& Ducibella 2006, Wassarman \& Litscher 2008). More recently, Dean and colleagues present evidence that the supramolecular structure of the $\mathrm{ZP}$, regulated by intactness of $\mathrm{mZP} 2$, is required for Al sperm binding (Rankin et al. 2003,
Gahlay et al. 2010). Once mZP2 is cleaved at Ala166 to form $\mathrm{mZP} 22_{\mathrm{f}}$, as occurring after fertilization (Burkart et al. 2012), the ZP matrix becomes refractory to sperm binding (Florman \& Ducibella 2006). Accumulated evidence also reveals the participation of acrosomal matrix proteins in ZP binding, a basis for how acrosome reacting sperm remain bound to the ZP (Kim \& Gerton 2003, Buffone et al. 2008a, 2008b). Proacrosin is an example of these acrosomal proteins, and it has affinity for both mZP2 and mZP3 (Howes et al. 2001). While all of these results confirm the significance of mZP3 and $\mathrm{mZP2}$ in sperm binding, they argue against the exclusiveness of mZP3 and mZP2 as primary and secondary sperm receptors respectively.

In contrast to the situation on the $\mathrm{ZP}$, a number of proteins on the sperm head have been described for ZP binding ability (Lyng \& Shur 2007, Tanphaichitr et al. 2007), and these observations are explained by the possibility that these proteins are backups for one another and/or act sequentially in gamete interaction. 
We have previously shown that sperm surface arylsulfatase A (ASA, EC 3.1.6.8) is involved in ZP binding in mice, pigs, and humans, and purified ASA can bind to intact or solubilized ZP directly (Rattanachaiyanont et al. 2001, Carmona et al. 2002a, Tantibhedhyangkul et al. 2002). Nonetheless, the identity of the ZP glycoproteins to which ASA binds was not known, and this was one specific aim of the current research study. We have postulated that ASA, as a sulfatase, interacts with sulfated sugar residues (Carmona et al. 2002a), which are components of the ZP glycans (Shimizu et al. 1983, Hirano et al. 1993, Noguchi \& Nakano 1993, Hokke et al. 1994, Takasaki et al. 1999, Dell et al. 2003, Topfer-Petersen et al. 2008, Pang et al. 2011). Our computational analyses indicate that the active site pocket of ASA is the positively charged area, which is essential for capturing the sulfated substrate (Lukatela et al. 1998, Schenk et al. 2009). Cys69, which is posttranslationally modified to become formylglycine, is the active site amino acid, but Lys123 and Lys302 present in the active site pocket are essential in capturing an arylsulfate substrate into the pocket (Lukatela et al. 1998, Schenk et al. 2009). Sulfogalactosylglycerolipid (SGG, aka seminolipid, present selectively and substantially in male germ cells) and sulfogalactosylceramide (SGC, present mainly in the brain and kidney) are known natural substrates of ASA, provided that the sulfoglycolipids are presolubilized into a micelle form by saposin B (Tanphaichitr et al. 2003). However, the ability of purified ASA to desulfate galactose sulfate has been documented (Waheed \& Van Etten 1980). Therefore, the second aim of our study was to determine whether Cys69, Lys123, and Lys302 in the active site pocket of ASA were important for ASA-ZP interaction. In addition, we wanted to verify whether ASA existed in the acrosome of mouse sperm, as documented in other mammalian sperm (Dudkiewicz 1984, Nikolajczyk \& O'Rand 1992, Brandon et al. 1997). Information of the
ASA localization on the sperm head should shed more light to its roles during sperm binding and penetration through the ZP.

\section{Results}

Binding of sperm to the egg ZP involves AI sperm in the initial step and acrosome reacting and $A R$ sperm in the subsequent stages. We have previously shown the presence of ASA (68 $\mathrm{kDa}$ ) on the convex ridge surface of the mouse Al sperm head (Tantibhedhyangkul et al. 2002). However, in other mammals ASA has also been shown as an acrosomal enzyme (Dudkiewicz 1984, Nikolajczyk \& O'Rand 1992, Brandon et al. 1997). Therefore, we asked whether mouse ASA was present as part of the acrosomal content (AC) and on the surface of AR sperm. A23187 was used to treat capacitated sperm to produce AR sperm along with the AC and acrosomal vesicles. Immunoblotting revealed the same anti-ASA reactive $68 \mathrm{kDa}$ band in $\mathrm{Al}$ and $\mathrm{AR}$ sperm, the $\mathrm{AC}$, and acrosomal vesicles (Fig. 1A). The $68 \mathrm{kDa}$ molecular weight of ASA observed herein in all of the four samples is similar to that previously described for Al sperm (Tantibhedhyangkul et al. 2002, Wu et al. 2007). Among the AR sperm, AC, and acrosomal vesicles samples, ASA existed at the highest level in the AC. The amount of ASA in the acrosomal vesicles (hybrid vesicles of the sperm head plasma membrane and outer acrosomal membrane) was the least; the $68 \mathrm{kDa}$ ASA band was apparent when the acrosomal vesicles sample was prepared from 10 million sperm. This was in contrast to the AC and AR sperm, where the $68 \mathrm{kDa}$ ASA band was already prominent from the samples prepared from 1 million sperm. Immunofluorescence was further employed to localize ASA on AR and Al sperm. OBF13, a mAb generated against the sperm membrane protein, Izumo (Kawai et al. 1989, Inoue et al. 2005), was used as a marker of AR sperm in this ASA localization. Izumo, a transmembrane protein, is localized over the entire head

A
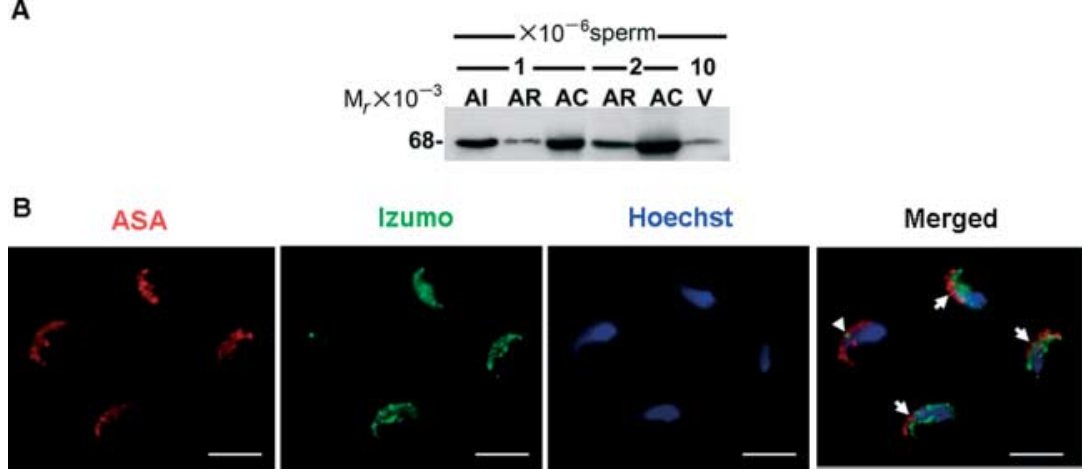

Figure 1 (A) Immunoblotting for ASA of proteins isolated from acrosome intact (AI) sperm, A23187-treated sperm with $\sim 80 \%$ acrosome reacted $(A R)$, acrosomal content (AC) and acrosomal vesicles (V). Proteins from 1 million Al sperm, 1 and 2 million AR sperm, from AC prepared from 1 and 2 million sperm, and from acrosomal vesicles prepared from 10 million sperm were used for gel loading. (B) Indirect immunofluorescence of ASA in AI and AR sperm. AR sperm were those that were positively labeled with OBF13 (green), a mAb against Izumo which is localized to the entire sperm head only after acrosome reaction. Note that ASA staining (red) was observed in the convex ridge of both AI (arrowhead) and AR (arrow). Blue staining from Hoechst denotes the sperm nucleus. The results shown are representative of two replicate experiments. Bar $=10 \mu \mathrm{m}$. 
of AR sperm and is exposed to react with OBF13 only after acrosomal exocytosis. The validity of identifying OBF13 stained sperm as AR sperm was first shown by comparing the number of AR sperm assessed by OBF13 reactivity with that obtained by Coomassie blue staining (a more widely used method; Bleil \& Wassarman 1990). Supplementary Figure S1 (see section on supplementary data given at the end of this article) indicates that the numbers acquired from the two methods were the same. Figure 1B shows the presence of ASA (red staining) on the convex ridge surface of an $\mathrm{Al}$ sperm (arrowhead), which had negative staining with OBF13. In AR sperm (arrows), which exhibited OBF13 staining (green), ASA was also present on the convex ridge surface; it was likely that ASA was localized on the inner acrosomal membrane of AR sperm. At the present time, the possibility that ASA is also present on the outer acrosomal membrane cannot be excluded.

Since ASA has direct binding ability to the mouse ZP (Tantibhedhyangkul et al. 2002), we asked whether ASA could bind selectively to mZP3 and mZP2 glycoproteins, both known as sperm receptors. These two mZP glycoproteins were prepared in quantity from isolated mouse ovarian ZP. Nonreducing SDS-PAGE/silver staining of solubilized $\mathrm{mZP}$ glycoproteins revealed the highest abundance of mZP2 followed by mZP3 and mZP1 (Fig. 2A, left lane) in agreement with the previous report (Green 1997). Size exclusion HPLC on a Bio-Sil SEC 250 column separated solubilized mouse ZP glycoproteins into three peaks with retention times of 57.3, 62.2, and $66.8 \mathrm{~min}$ (Fig. 2C). SDS-PAGE with silver staining revealed these three peaks in the elution order to be mZP1 (200 kDa - dimeric form), mZP2 (120 kDa), and mZP3 (83 kDa) (Fig. 2A). The identity of the isolated mZP2 and mZP3 was further revealed by their specific reactivity to the monoclonal anti-mZP2 and anti-mZP3 antibody respectively (Fig. $2 \mathrm{~B}$ ). We further showed that the isolated $\mathrm{mZP} 3$, but not $\mathrm{mZP2}$, was able to induce the sperm acrosome reaction, as previously described (Wassarman \& Litscher 2008; Fig. 2D). The yield of mZP1, mZP2, and mZP3 was about 35, 200, and 150 ng/ovary, respectively. The weight ratio of the purified $\mathrm{mZP2}$ to mZP3 (1:0.75) was similar to that previously described (1:0.7) (Green 1997), although the ratio of the obtained mZP1 to that of mZP2 $(0.18: 1)$ was lower than the described value (0.3:1). Subsequently, mZP2 and mZP3 were also purified by electroelution from the polyacrylamide gel of total solubilized ZP glycoproteins that were subjected to SDS-PAGE. The yield of electroeluted mZP3 was comparable to that obtained by HPLC, although the efficiency to prepare mZP2 by electroelution was lower than the HPLC method (see Supplementary Table S1, see section on supplementary data given at the end of this article).

To demonstrate direct affinity of ASA to $\mathrm{mZP}$ glycoproteins, mZP1, mZP2, and mZP3 were individually immobilized onto the nitrocellulose membrane.
Total solubilized mZP (1 $\mu \mathrm{g}$, $\sim 9$ pmol using $110 \mathrm{kDa}$ as the average molecular weight of the whole mZP glycoproteins), previously shown for ASA affinity, served as a positive control, whereas ovalbumin $(1 \mu \mathrm{g})$ without ASA binding ability (Tantibhedhyangkul et al. 2002) was
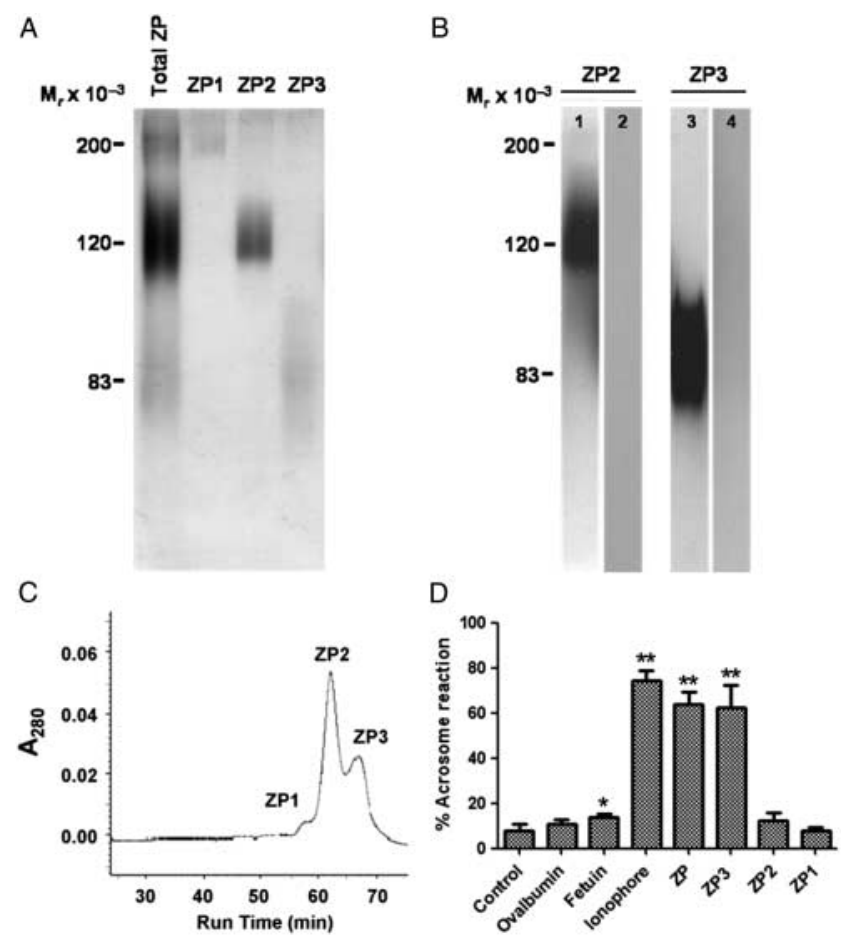

Figure 2 Isolation and characterization of purified ZP glycoproteins. (A) Nonreducing SDS-PAGE of ovarian mouse ZP. The isolated zonae pellucidae were solubilized in $0.2 \mathrm{M}$ sodium phosphate buffer, $\mathrm{pH}$ 6.8, containing $0.1 \%$ SDS by heating at $65^{\circ} \mathrm{C}$ for $10 \mathrm{~min}$ and then subjected to SDS-PAGE followed by silver staining (total ZP lane). Two

micrograms of solubilized total ZP were used for gel loading. For ZP1, $Z P 2$, and ZP3, the amount loaded was equivalent to that purified from the solubilized total ZP of $2 \mu \mathrm{g}$. The apparent molecular masses of mZP1, mZP2, and mZP3 were 200, 120, and $83 \mathrm{kDa}$ respectively. (B and C) Fractionation of solubilized mouse ZP by HPLC on a Bio-Sil SEC 250 size-exclusion column. The chromatogram (C) shows the separated fractions of $\mathrm{mZP} 1, \mathrm{mZP} 2$, and $\mathrm{mZP} 3$, which had distinctive electrophoretic mobility by nonreducing SDS-PAGE (ZP1, ZP2, and ZP3 lanes in A panel). The identity of the $\mathrm{mZP} 2$ and $\mathrm{mZP} 3$ fractions was further confirmed by immunoblotting (B). mZP2 (lanes 1 and 2) and mZP3 fractions (lanes 3 and 4) (0.5 $\mu$ g each) were probed with both anti-ZP2 (lanes 1 and 4) and anti-ZP3 (lanes 2 and 3). (D) Ability of solubilized mouse ovarian ZP and purified mZP3 to induce the acrosome reaction. Capacitated sperm were incubated with solubilized mouse ZP, and purified mZP1, mZP2, and mZP3 at a final concentration of $8 \mathrm{ZP} / \mu \mathrm{l}$ or an equivalent. Sperm treated with $10 \mu \mathrm{M}$ A23187 served as a positive control. Sperm were also treated with ovalbumin $(40 \mathrm{ng} / \mu \mathrm{l})$ and fetuin $(40 \mathrm{ng} / \mu \mathrm{l})$. Only total solubilized mZP and $\mathrm{mZP} 3$ could induce the acrosome reaction in a similar capacity to A23187, and the levels of AR sperm produced were highly significantly different from control untreated sperm $(P<0.005$, as analyzed by Student's $t$-test and denoted by $\left.{ }^{* *}\right)$. mZP1 and mZP2 and ovalbumin did not induce acrosome reaction. Fetuin slightly increased the number of AR sperm ( $\sim 8 \%$ with $P<0.05 \%\left({ }^{*}\right)$ as compared with the untreated control sperm sample). Data were expressed as mean \pm s.D. from three replicate experiments. 
used as a negative control. Fetuin, a serum glycoprotein, which is anionic due to sialylation of its carbohydrate moieties (Takasaki \& Kobata 1986), was also determined for its affinity for ASA. Three micrograms of purified ASA (44 pmol, $\sim 5 \times$ mole ratio to the mZP glycoprotein dot) were used for all mZP glycoprotein dot incubation. In the first set of experiments, the proportion of the three mZP glycoproteins used for this immobilization mimicked that present in the total solubilized ZP (i.e. $0.3 \mu \mathrm{g}$ for $\mathrm{mZP} 1,1 \mu \mathrm{g}$ for $\mathrm{mZP} 2$, and $0.7 \mu \mathrm{g}$ for mZP3; Green 1997). At this weight ratio of the three mZP glycoproteins, ASA bound to mZP2 at the highest level, followed by mZP3, whereas the binding of ASA to mZP1 was at a background level as observed with ovalbumin (Fig. 3A). The order of the ASA affinity for the three mZP glycoproteins was still observed when the three mZP glycoproteins were compared at the same weights of 0.3 and 0.7 , and $1 \mu \mathrm{g}$. However, mZP1 also bound to ASA but at the lowest level (Fig. 3C and D). The levels of ASA binding to $0.1 \mu \mathrm{g}$ mZP2 and mZP1 glycoproteins appeared to be minimal and similar to each other, suggesting that these amounts of mZP2 and mZP1 glycoproteins may be under the limit of detection for their ASA binding (Fig. 3C and D). The binding of ASA to mZP2 and mZP3 appeared to reach a plateau level already at $0.7 \mu \mathrm{g}$ of the two mZP glycoproteins, suggesting that the ASA-mZP2/mZP3 interaction was specific. Further, preincubation of ASA with $10 \mu \mathrm{g}$ of solubilized $\mathrm{mZP}$ glycoproteins $(5 \times$ and $10 \times$ mole ratio to $\mathrm{mZP} 2$ and $\mathrm{mZP} 3$, immobilized onto the nitrocellulose membrane respectively) abolished its affinity to both mZP glycoproteins (Fig. 3B). In addition, we showed that ASA did not bind to another anionic glycoprotein, fetuin, suggesting that ASA-ZP glycoprotein binding is a specific phenomenon (Fig. 3A).

ASA is a sulfatase with known desulfation activity for two natural sulfoglycolipids, SGG and SGC (Hanson et al. 2004, Schenk et al. 2009, Xu et al. 2011), each expressing galactose sulfate as their head group. Galactose sulfate has also been shown to be desulfated by purified ASA (Waheed \& Van Etten 1980). Since sulfated sugar residues are part of the ZP glycans (Shimizu et al. 1983, Hirano et al. 1993, Noguchi \& Nakano 1993, Hokke et al. 1994, Takasaki et al. 1999,
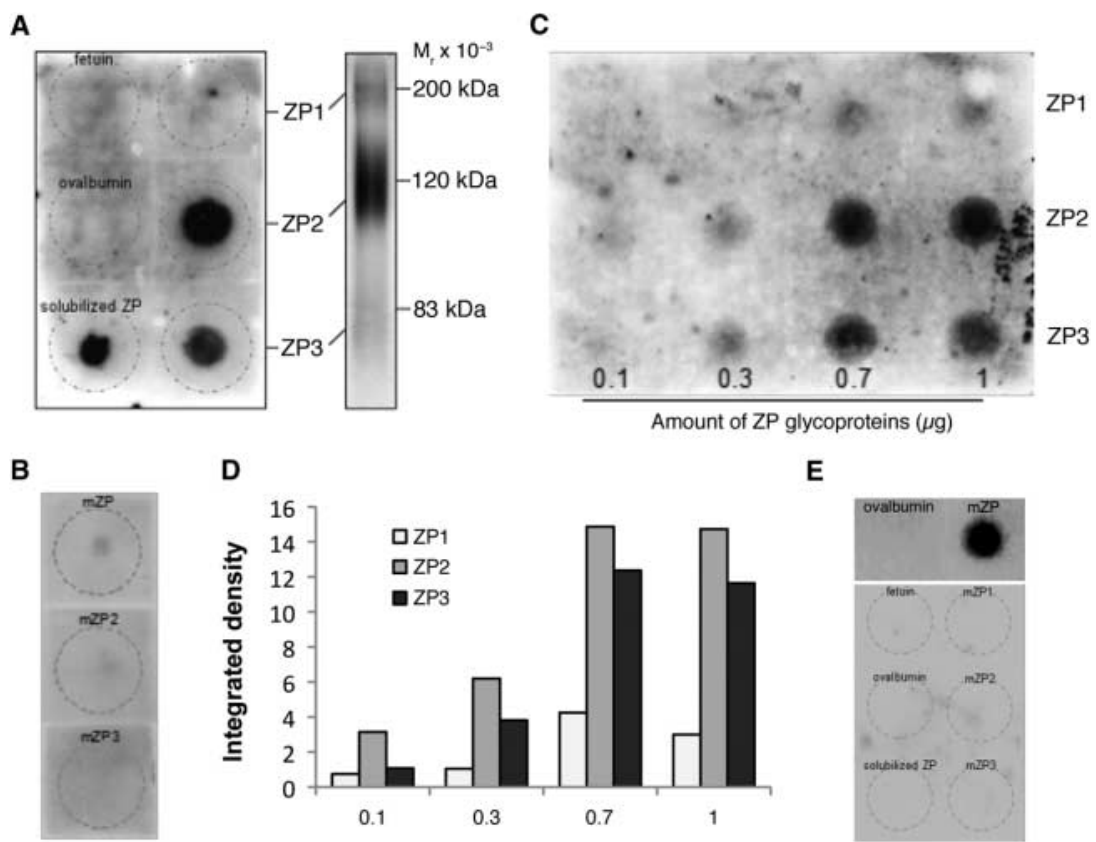

D

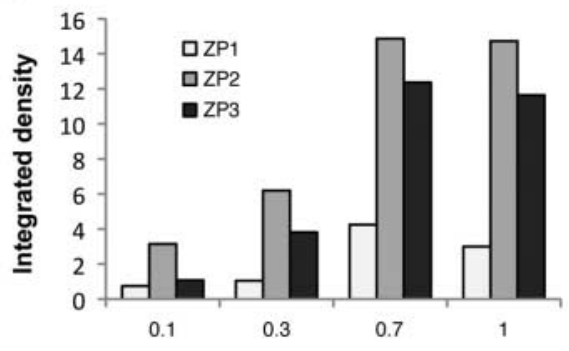

E

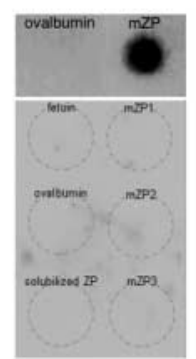

Figure 3 Binding of purified sperm ASA to solubilized mouse ovarian ZP and individual ZP glycoproteins. (A, B, C and D) ASA had affinity for mZP2 and mZP3. (A) One microgram of fetuin, ovalbumin, and solubilized whole mZP glycoproteins, $0.3 \mu \mathrm{g}$ of $\mathrm{mZP} 1,1 \mu \mathrm{g}$ of $\mathrm{mZP} 2$, and $0.7 \mu \mathrm{g}$ of $\mathrm{mZP} 3$ were dotted onto nitrocellulose membrane. The weight ratio of mZP1, mZP2, and mZP3 used for the dotting was as described previously (Green 1997). After incubation with purified ASA ( $3 \mu \mathrm{g})$, the membrane was washed extensively to remove unbound protein and probed for the ASA binding by anti-ASA antibody and HRP-conjugated secondary antibody. (B) One microgram each of mZP, mZP2, and mZP3 was immobilized onto the nitrocellulose membrane. ASA was preincubated with $10 \mu \mathrm{g}$ of solubilized mZP prior to the incubation with the membrane. Note that solubilized $\mathrm{mZP}$ saturated the ZP binding site(s) on ASA, thus preventing it from interacting with $\mathrm{mZP}, \mathrm{mZP} 2$, and mZP3 immobilized onto the nitrocellulose membrane. (C) Various amounts of mZP1, mZP2, and mZP3 were immobilized onto the nitrocellulose membrane, which was then incubated with ASA. The incubation conditions and the detection of ASA-ZP binding were as described in (A). (D) Densitometric analyses of the binding of ASA to $\mathrm{mZP} 1, \mathrm{mZP} 2$, and $\mathrm{mZP} 3$. Note that mZP2 bound to ASA to the highest degree followed by mZP3. The binding of ASA to mZP1 was markedly lower. (E) Binding of ASA to mouse zona glycoproteins was inhibited by dextran sulfate (lower panel) but not by dextran (upper panel). Total mZP glycoproteins, mZP1, mZP2, mZP3, ovalbumin, and fetuin were dotted onto the nitrocellulose membrane as in (A). The conditions of ASA incubation and detection of the binding were also the same as in (A), except that the ASA incubation was performed in the presence of $1 \mu \mathrm{M}$ of dextran sulfate or dextran. All the results were representative of two or three replicate experiments. 


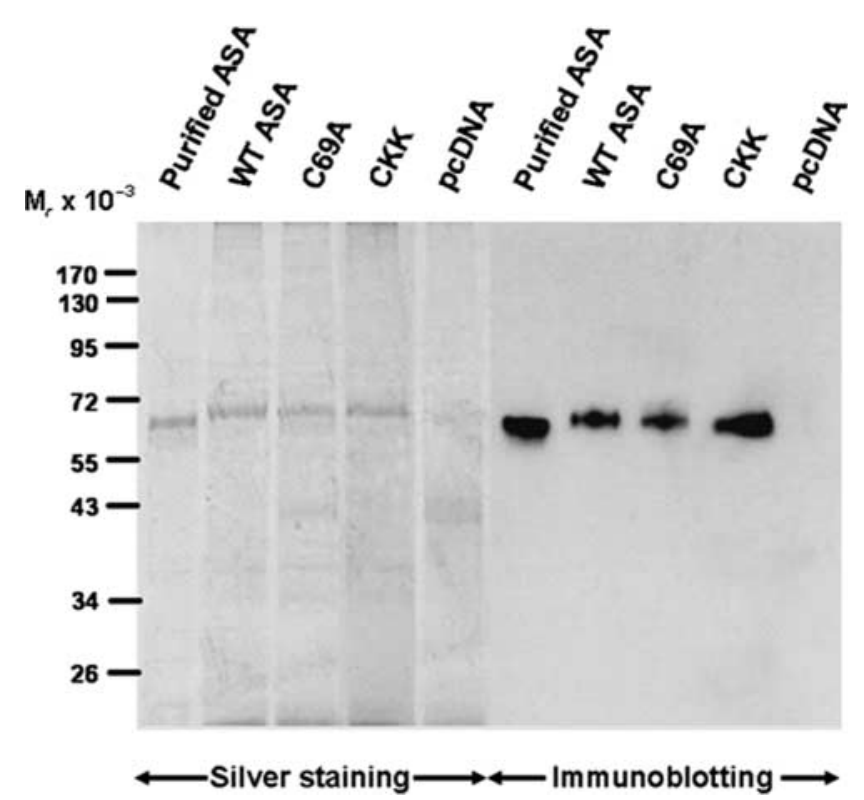

Figure 4 Purity of native ASA and recombinant WT, C69A, and CKK mutant ASAs. Native ASA was purified from pig sperm. All recombinant ASAs were produced in CHO-K1 cells and were directed to be secreted into the medium. Recombinant ASAs were purified as described in Materials and Methods. Medium from 'sham' transfection (using uninserted pcDNA3.1) was also collected for parallel purification (pcDNA). Left panel: SDS-PAGE/silver staining of native ASA (purified ASA), WT recombinant (rec) ASA, C69A (rec ASA with C69 substituted with Ala), CKK (rec ASA with C69, K123, and K302, all substituted with Ala), and pcDNA; right panel: corresponding immunoblotting with anti-ASA antibody. The same amount of protein after the final purification step (50 ng) was used for gel loading for all samples except for pcDNA, which was loaded based on an equivalent volume of the sample at the final step of purification. Note the purity of all rec ASAs, which appeared as a single major band on the silver stained gel.

Dell et al. 2003, Topfer-Petersen et al. 2008, Pang et al. 2011), it is possible that the interaction between ASA and ZP glycoproteins may be via the binding of ASA to sulfated sugars on the ZP. This prompted us to test whether dextran sulfate, a long-chain polymer of sulfated glucose, could inhibit ASA-ZP binding. Figure $3 \mathrm{E}$ (lower panel) reveals that binding of ASA to total solubilized mZP glycoproteins was minimal in the presence of $1 \mu \mathrm{M}$ dextran sulfate. However, dextran of the same concentration did not inhibit ASA-ZP interaction (Fig. 3E, upper panel). Therefore, the results suggested that sulfation of the ZP glycans may be important for the ASA-ZP interaction.

We have shown previously that the active site pocket of the three-dimensional (3D) structure of ASA is the positively charged area to which SGG and SGC and an artificial substrate, $p$-nitrocatechol sulfate (NCS), would readily dock (Schenk et al. 2009). In this active site pocket, Cys69, which is posttranslationally modified to be formylglycine, is the active site amino acid responsible for the removal of the sulfate group from the substrate. Lys123 and Lys302, present also in the active site pocket, are the positively charged amino acids that are mainly responsible in capturing sulfated sugar residue of the substrate in this active site pocket (Schenk et al. 2009). It can be speculated that the active site pocket of ASA is important in capturing the ZP glycan via its interaction with the sulfated sugar residue of the ZP. The mutation of Cys69 to Ala prevents ASA's desulfation activity and should thus prolong the binding of the sulfated sugar to the active site pocket. In contrast, mutant ASA, with Cys69, Lys123, and Lys302 substituted with Ala (CKK), was expected to have reduced binding to ZP glycoproteins, if the active site pocket of ASA is important for ZP glycan interaction. Figure 4 (left side) shows that partially purified rec wild type (WT) ASA, and the two mutant ASAs, C69A and CKK, appeared as a major band of about $70 \mathrm{kDa}$ molecular mass upon SDSPAGE/silver staining, and they were recognized by antiASA antibody (Fig. 4, right side). The 'sham' purification sample from CHO-K1 cells transfected with pcDNA did not possess the ASA band. It was noted that all rec ASAs had a slightly slower mobility than native ASA purified from pig sperm (Fig. 4, left side). It was possible that the glycosylation of rec ASA in $\mathrm{CHO}-\mathrm{K} 1$ cells was at a higher level, as compared with that in pig reproductive cells. As expected, rec WT ASA contained desulfation activity on NCS and its specific activity increased 7.6-fold after its purification using the Mono-Q-Sepharose resin. In contrast, the two mutant ASAs, C69A and CKK, had minimal NCS desulfation activity, slightly above the background value of the pcDNA sample (Table 1). However, the ability of rec WT and the two mutant ASAs to bind to the solubilized ZP immobilized onto nitrocellulose membrane was at similar levels (Fig. 5), a result suggesting that the active site pocket of ASA was not essential for the ZP binding.

\section{Discussion}

We have shown previously that ASA on the plasma membrane of the mouse, human, and pig sperm head is

Table 1 Desulfation activity of recombinant wild type and mutant ASAs. CHO-K1 cells transfected with full-length ASA cDNA, C69A construct, C69A/K123A/K302A (CKK) construct, and empty vector pcDNA3.1 were treated with $10 \mathrm{mM}$ ammonium chloride in serum-free culture medium to induce secretion of newly synthesized ASA. The spent culture media were collected and an aliquot was used for protein quantification and NCS desulfation activity assay (medium). ASA in the remaining media was further purified using Q-sepharose resin and the total protein obtained and NCS desulfation activity was measured (after QS).

\begin{tabular}{lcccc}
\hline & WT & C69A & CKK & pcDNA \\
\hline Medium & & & & \\
$\quad$ Protein (mg) & 3.67 & 5.51 & 6.69 & 6.76 \\
Total activity (U) & 17.90 & 2.90 & 2.42 & 1.94 \\
Specific activity (U/mg) & 4.88 & 0.53 & 0.36 & 0.29 \\
After QS & & & & \\
Protein (mg) & 0.57 & 1.98 & 1.12 & 2.79 \\
Total activity (U) & 21.1 & 2.65 & 0.65 & 0.68 \\
Specific activity (U/mg) & 37.02 & 1.34 & 0.58 & 0.24 \\
\hline
\end{tabular}



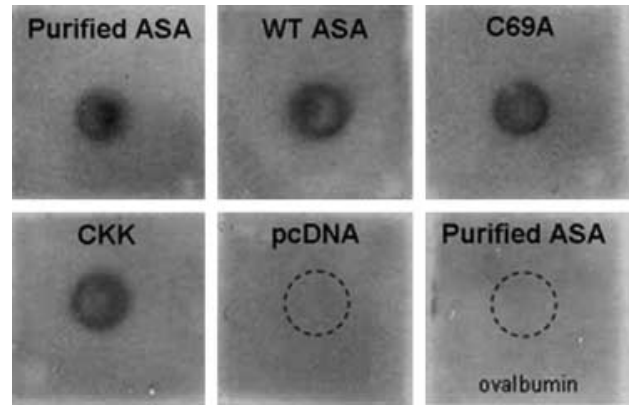

Figure 5 Binding of recombinant mutant ASAs to solubilized mouse ovarian ZP. One microgram of solubilized mZP glycoproteins or ovalbumin (labeled 'ovalbumin' in the bottom right corner panel), dotted onto the nitrocellulose membrane, was incubated with $1 \mu \mathrm{g}$ of native purified ASA, rec WT ASA and mutant ASAs C69A, and CKK. An equivalent volume of pcDNA sample from the final purification step was also used for incubation. Note that all types of ASAs bound at a similar level to solubilized mZP, but no binding was observed with ovalbumin or when pcDNA was used in place of ASA for the incubation. The results were representative of two replicate experiments.

involved in sperm-ZP interaction and purified ASA binds to both intact and solubilized ZP (Rattanachaiyanont et al. 2001, Carmona et al. 2002a, Tantibhedhyangkul et al. 2002, Wu et al. 2007). In this report, we provided evidence that ASA was also present in the mouse AC and on the inner acrosomal membrane (Fig. 1) and it had a preferential binding ability to mZP2 and mZP3. Like ASA on the sperm plasma membrane (Weerachatyanukul et al. 2003), ASA may be anchored onto the inner acrosomal membrane via its affinity for SGG (Carmona et al. 2002b), which also exists on this surface membrane (Supplementary Figure S2, see section on supplementary data given at the end of this article). Our finding on the presence of ASA as an AC component is in agreement with the previous reports in other mammalian sperm (Dudkiewicz 1984, Nikolajczyk \& O'Rand 1992, Brandon et al. 1997). It is still unclear whether ASA in the AC binds to any ligands. Notably, ASA in the AC was of the highest quantity as compared with ASA on AR sperm and acrosomal vesicles (hybrid membranes between the sperm head plasma membrane and outer acrosomal membrane; Fig. 1A). ASA itself oligomerizes forming a dimer at neutral $\mathrm{pH}$ and an octamer at $\mathrm{pH} 5$ (Lukatela et al. 1998). Since the intra-acrosomal $\mathrm{pH}$ is between 5.3 and 6.2 (Nakanishi et al. 2001), ASA in the $\mathrm{AC}$ should exist in the oligomeric form of a size in between a dimer and an octamer. The oligomeric forms of ASA, like those of sp56, another acrosomal protein with ZP binding ability, would likely exist as scaffolds beneficial for the interaction between acrosome reacting sperm and the ZP (Buffone et al. 2008a, 2008b).

Previous studies of Wassarman and colleagues. have indicated that $\mathrm{mZP} 3$ is the primary sperm receptor, binding to $\mathrm{Al}$ sperm, whereas mZP2 acts as the secondary receptor, interacting with $A R$ sperm (Bleil et al. 1988, Wassarman \& Litscher 2008). Our results indicating the binding ability of ASA, which exists on the sperm head plasma membrane, in the $A C$ and on the inner acrosomal membrane to both $\mathrm{mZP} 2$ and $\mathrm{mZP} 3$ challenge the sharp demarcation of $\mathrm{mZP} 3$ and $\mathrm{mZP} 2$ as a receptor of $\mathrm{Al}$ and $\mathrm{AR}$ sperm respectively. In fact, more recent work indicates that both purified $\mathrm{mZP} 3$ and $\mathrm{mZP} 2$ can bind to both Al and AR sperm (Kerr et al. 2002). Furthermore, the supramolecular structure model of Dean et al. argues that sperm would interact with both $\mathrm{mZP} 3$ and $\mathrm{mZP} 2$, provided that $\mathrm{mZP} 2$ is intact; only after a cleavage of $\mathrm{mZP} 2$ by a cortical granule protease to generate $\mathrm{mZP}_{\mathrm{f}}$ (Burkart et al. 2012), sperm can no longer bind to the ZP (Gahlay et al. 2010). Supporting the argument that the binding of mZP2 and mZP3 to sperm is a continuous event are the findings that a number of acrosomal proteins bind to the ZP (e.g. proacrosin, sp56, zonadhesin, sp38), and proacrosin, like ASA, has the affinity for both mZP2 and mZP3. Electron microscopy immunogold labeling also indicates that $\mathrm{mZP} 2$ and $\mathrm{mZP} 3$ are localized next to each other on the ZP (El Mestrah et al. 2002), and both of these ZP glycoproteins have the propensity to form fibrils (Greve \& Wassarman 1985, Litscher et al. 2008, Jimenez-Movilla \& Dean 2011). The ability of sperm proteins, including ASA (this report) and proacrosin (Howes et al. 2001), to bind to both mZP2 and mZP3 should give enhanced interaction between sperm and the ZP. The binding ability of $\mathrm{mZP} 2 / \mathrm{mZP} 3$ fibrils, as compared with solubilized $\mathrm{mZP} 2$ and $\mathrm{mZP} 3$, to sperm should be the matter of the future investigation.

Mammalian ZP glycans contain sulfated sugar residues, and their significance in sperm binding has been implicated (Shimizu et al. 1983, Oehninger et al. 1991, Mori et al. 1993, Yonezawa et al. 1995, Howes et al. 2001, Howes \& Jones 2002, Moreno et al. 2002, Dell et al. 2003, Gaboriau et al. 2007, Lin et al. 2007, Topfer-Petersen et al. 2008, Yu et al. 2009, Pang et al. 2011). A number of sulfatases have desulfation activity on sulfated sugar residues (Hanson et al. 2004). Although ASA is better known for its desulfation activity on sulfoglycolipids (SGG and SGC; Schenk et al. 2009), the ability of ASA to desulfate galactose sulfate residues has also been documented (Waheed \& Van Etten 1980). The binding of sulfated sugar residues on the ZP glycans to the active site pocket of ASA may thus be a basis of ASA$Z P$ interaction. Results revealing that dextran sulfate, but not dextran, could inhibit ASA-ZP2/ZP3 interaction (Fig. 3E) indicated the involvement of sulfated glycans in this interaction. However, ASA-ZP2/ZP3 interaction was still retained when the key amino acids that capture the sulfated substrate in the active pocket (i.e. Cys69, Lys123, and Lys302) were all mutated to Ala (the so-called CKK mutant ASA; Fig. 5), indicating that the ZP glycans did not bind to ASA's active site pocket. Our previous computational modeling for electrostatic potentials of ASA reveals that the active site pocket is 
the highest for positive charges, although it is not well exposed. Positively charged areas are also found on certain areas on the surface of ASA's 3D structure (Schenk et al. 2009). Computational docking shows that SGG/SGC can also fit into a surface cleft consisting of Arg496, a positively charged amino acid, and Ile487, a hydrophobic amino acid (Supplementary Figure S3, see section on supplementary data given at the end of this article). This ASA-SGG interaction is likely a basis on how ASA from the epididymal fluid deposits onto the head convex ridge of transiting sperm without subsequent SGG hydrolysis (Carmona et al. 2002b). Likewise, ASA synthesized in spermatogenic cells and targeted to the acrosomal process may remain on the inner acrosomal membrane (Fig. 1B) via affinity for SGG at this R496/1487 site. ASA at neutral $\mathrm{pH}$ exists as a dimer and at acidic $\mathrm{pH}$ as a higher-ordered oligomer (Lukatela et al. 1998). Only one ASA molecule in the dimer or oligomer may bind to SGG on the sperm membrane, allowing the R496/I487 site on the other ASA molecule(s) for interaction with sulfated sugar residues of the ZP glycans. In addition, other surface positively charged amino acids may participate in binding to sulfated ZP glycans. In fact, we have previously shown that purified ASA can bind to chondroitin sulfate B glycosaminoglycans (disaccharide repeats of iduronic acid - N-acetylgalactosamine-4-sulfate) in a nonenzymatic manner (Wu et al. 2007). However, the computational docking of a long-chain glycan such as the ZP glycan to a protein is not practical and likely to be inaccurate due to the high flexibility of the glycan chain. In addition, information on the primary sequence of sulfated mZP glycans as well as higher-ordered structures of ZP glycans is not known. The latter piece of information may be relevant to ASA binding, considering that $\mathrm{mZP} 1$ is sulfated to the same extent as $\mathrm{mZP2}$, but $\mathrm{mZP} 1$, in contrast to $\mathrm{mZP} 2$, showed only minimal binding ability to purified ASA (Fig. 3).

Since the binding of the ZP to ASA was not dependent on the active site pocket of the enzyme, ASA should still contain desulfation activity, which may be relevant during fertilization. It may desulfate SGG released as part of the acrosomal vesicles from the sperm head plasma membrane, provided that saposin B, the co-factor of ASA is available to 'solubilize' SGG from acrosomal vesicles and deliver the sulfoglycolipid into ASA's active site pocket (Ahn et al. 2003). This is possible, since prosaposin is present in the oviductal fluid (M Schenk, DC Santos and N Tanphaichitr, unpublished observations) and cathepsin D, which is known for its processing activity of prosaposin to saposin B (Hiraiwa et al. 1997), is also released as part of the AC (Erickson \& Martin 1974). This SGG desulfation may be essential; acrosomal vesicles would still adhere to the ZP due to SGG affinity for the ZP (White et al. 2000), thus impeding sperm migration through the ZP layer. On the other hand, galactosylglycerolipid (GG), the desulfated product of SGG, has minimal affinity for the ZP (White et al. 2000) and would thus be released into the surrounding. We are currently investigating these possibilities to gain a better understanding of ASA-ZP interaction. Regardless, our results herein showed that ASA bound preferentially to $\mathrm{mZP} 2$ and $\mathrm{mZP} 3$, the known mouse sperm receptors, and was localized on the convex ridge head surface of both Al and AR sperm as well as in the AC. All these properties implicate that sperm ASA has physiological significance in ZP binding.

\section{Materials and Methods}

\section{Animal use}

Sexually mature CF- 1 female mice (6-8 weeks of age) and CD-1 male mice ( $\sim 10$ weeks of age) were purchased from Charles Rivers (Montreal, QC, Canada), and were housed in temperature and humidity controlled rooms with $14 \mathrm{~h}$ light:10 $\mathrm{h}$ darkness cycles. They were provided with food and water ad libitum. Both male and female mice were killed by cervical dislocation. The use of all mice was in accordance to the guidelines of the Canadian Council on Animal Care, as approved by the Animal Care Committee of OHRI.

\section{Immunolocalization of ASA on AI and AR sperm}

Mouse sperm were collected from the cauda epididymis and vas deferens in the KRB medium and subjected to Percoll gradient centrifugation (PGC), following our described method (Tantibhedhyangkul et al. 2002). PGC Al sperm were capacitated $\left(30 \mathrm{~min}, 5 \% \mathrm{CO}_{2}, 37^{\circ} \mathrm{C}\right.$ ) in $\mathrm{KRB}-0.3 \% \mathrm{BSA}$, and then treated with $10 \mu \mathrm{M}$ A23187 as previously described (lamsaard et al. 2011). This treatment induced acrosome reaction in $70-80 \%$ of the total sperm population. Localization of ASA in $\mathrm{Al}$ and AR sperm was performed by immunofluorescence with the concurrent use of mouse mAb OBF13 IgM (recognizing the sperm membrane protein, Izumo, only in AR sperm (Kawai et al. 1989, Inoue et al. 2005), a gift from Dr Masaru Okabe, Osaka University) to differentiate AR from Al sperm. The A23187-treated sperm were washed twice with PBS by centrifugation ( $350 \mathrm{~g} 10 \mathrm{~min}$, room temperature (RT)) and blocked with 5\% de-complemented normal goat serum (prepared in PBS) for 15 min at RT. After washing with PBS by centrifugation, the sperm were incubated (30 min, RT) with $10 \mu \mathrm{g} / \mathrm{ml}$ rabbit polyclonal anti-ASA IgG (produced and purified for the IgG fraction as described (Wu et al. 2007)) plus $0.5 \mu \mathrm{g} / \mathrm{ml}$ OBF13 IgM in PBS. Following washing with PBS by centrifugation, the sperm were incubated ( $30 \mathrm{~min}, \mathrm{RT})$ with secondary antibodies, $25 \mu \mathrm{g} / \mathrm{ml}$ Alexa 594-conjugated goat anti-rabbit IgG and $5 \mu \mathrm{g} / \mathrm{ml}$ Alexa 488-conjugated goat antimouse IgM (Invitrogen). Sperm treated with preimmune rabbit serum IgG and normal mouse IgM in place of the primary antibodies served as negative controls. To assess cell viability during the staining process, propidium iodide (Invitrogen) was added to the cell suspension at a final concentration of $1 \mu \mathrm{g} / \mathrm{ml}$ 5 min before the secondary antibody incubation period ended. Hoechst 33342 (Invitrogen, at the final concentration of $0.5 \mu \mathrm{g} / \mathrm{ml}$ ) was also added at the same time as propidium iodide to stain the sperm nucleus. The sperm were then washed twice in PBS, mounted onto a slide in 50\% PBS/50\% glycerol 
and viewed under a Zeiss IM35 epifluorescence microscope (Carl Zeiss Canada, Toronto, ON, Canada). Fluorescent images of sperm were recorded by a Spot Junior CCD camera (Carl Zeiss Canada).

\section{Isolation of AI capacitated sperm, AR sperm, acrosomal vesicles, and $A C$ for immunoblotting}

The preparation of $\mathrm{Al}$ capacitated and $\mathrm{AR}$ sperm was as described above. However, in the immunoblotting detection of ASA, which had a molecular mass $(68 \mathrm{kDa})$ similar to BSA present in the capacitation medium, the high amount of BSA remaining in the supernatant of A23187-treated sperm could cause distortion of the ASA-reactive band, especially in the AC fraction. Therefore, the Al capacitated sperm were prewashed in KRB supplemented with $0.01 \%$ BSA prior to A23187 treatment (lamsaard et al. 2011). Acrosomal vesicles and AC were prepared from the supernatant of A23187-treated sperm by ultracentrifugation as previously described (lamsaard et al. 2011); acrosomal vesicles sedimented as a pellet and $A C$ remained in the supernatant. $\mathrm{AI}$ and $\mathrm{AR}$ sperm (1 million each) and AC and acrosomal vesicles isolated from 1 million A23187-treated sperm were subjected to SDS-PAGE and immunoblotting for ASA.

\section{Purification of native ASA and production of recombinant $W T$ and mutant $A S A$}

Native ASA was purified from boar sperm following the described method (Wu et al. 2007). The purified ASA had a specific activity of $\sim 60 \mathrm{U} / \mathrm{mg}$ (assayed with NCS as a substrate), and appeared as a single band on a $10 \%$ polyacrylamide gel following SDS-PAGE/silver staining (see below).

Recombinant (rec) WT ASA and C69A and CKK mutant ASAs were produced in Chinese hamster ovary-K1 (CHO-K1) cells, following transient transfection of the cells with pcDNA3.1 expression vector containing WT and mutant sequences of $A S A$, as previously described (Schenk et al. 2009). Although the ASA sequence in the pcDNA3.1 plasmid was of the pig species, pig and mouse ASA sequences bear 96\% conserved homology. Further, purified pig ASA binds specifically to mouse intact ZP (Tantibhedhyangkul et al. 2002). C69A was the mutant ASA that had the active site amino acid, Cys69, mutated to Ala. For CKK, Cys69 and the two lysine residues, Lys123 and Lys302, important for capturing the sulfate group of the substrate, were substituted with Ala. By culturing the transfected cells in medium containing $10 \mathrm{mM}$ ammonium chloride for $18 \mathrm{~h}$, ASA, which is a lysosomal enzyme, was secreted into the medium. The spent medium was collected for rec ASA purification, following our described method (Schenk et al. 2009), which involved size exclusion dialysis, Q-Sepharose resin capturing, and size exclusion Microcon YM-100 (Millipore Canada Ltd, Etobicoke, ON, Canada) centrifugation. However, in this current preparation, an additional step was included to remove transferrin (a CHO-K1 medium supplement) from rec ASA. Anti-transferrin IgG (Sigma) was coupled with Aminolink beads (Pierce, Rockford, IL, USA), following the manufacturer's instruction. The Microcon YM-100 retentate containing rec
ASA + transferrin $(\sim 100 \mu \mathrm{g})$ was then incubated with $30 \% \mathrm{v} / \mathrm{v}$ antibody-coupled Aminolink beads in a total volume of $\sim 600 \mu \mathrm{l}$ Tris-buffered saline (TBS: $137 \mathrm{mM} \mathrm{NaCl}$ in $20 \mathrm{mM}$ Tris- $\mathrm{HCl}, \mathrm{pH}$ 7.6) for $2 \mathrm{~h}$ at $4{ }^{\circ} \mathrm{C}$ with gentle shaking. After centrifugation $(3000 \mathrm{~g}, 1 \mathrm{~min}, \mathrm{RT})$, the supernatant, containing mainly recombinant ASA, was collected. The pelleted beads were washed three times with $0.4 \mathrm{ml}$ of TBS, $\mathrm{pH} 7.4$, and all three washes were collected and combined with the supernatant. The combined samples were further concentrated in TBS with a Microcon YM-50. Aliquots of the final product were assayed for NCS desulfation activity and subjected to SDSPAGE and immunoblotting.

\section{SDS-PAGE and immunoblotting for ASA and $m Z P$ glycoproteins}

Proteins were resolved by SDS-PAGE according to Laemmli (1970). For detection of ASA (native and rec ASA, AI and AR sperm, AC and acrosomal vesicles) the proteins were electrophoresed under a reducing condition in a $10 \%$ polyacrylamide gel $(0.75 \mathrm{~mm}$ thickness $)$. On the other hand, a nonreducing condition was used in electrophoresing mZP glycoproteins on a $7 \%$ polyacrylamide gel ( $1.5 \mathrm{~mm}$ thickness). Protein detection was by silver staining using a Bio-Rad Silver Stain kit.

Proteins separated on gel were transelectrophoresed (250 mA, 1 h) onto nitrocellulose membrane $(0.45 \mu \mathrm{m}$; Towbin \& Gordon 1984) for immunoblotting and chemiluminescence detections. For ASA, our previously described method was followed (Wu et al. 2007). For $\mathrm{mZP} 2$ and $\mathrm{mZP} 3$, rat monoclonal anti-mZP3 IgG and anti-mZP2 IgG antibodies were used (East et al. 1984, 1985). IgG fraction of rat anti-mZP3 and anti-mZP2 was purified, using a Protein G column kit from Thermo Scientific (Rockford, IL, USA), from the spent medium of hybridomas, which produced these mAbs (gifts from Dr J Dean, NIH). Nonspecific binding sites on the membrane were blocked ( $1 \mathrm{~h}, \mathrm{RT}$ ) with $5 \%$ nonfat milk in TBS containing $0.05 \%$ Tween 20 (TBST). The membrane was then incubated ( $1 \mathrm{~h}, \mathrm{RT}$ or $4{ }^{\circ} \mathrm{C}$, overnight) with $5 \mu \mathrm{g} / \mathrm{ml}$ of rat anti-mZP3 IgG or antimZP2 IgG, washed three times ( 5 min each) with TBST and then incubated with goat HRP-conjugated anti-rat IgG (Santa Cruz Biotechnology, Santa Cruz, CA, USA; 1:1000 dilution in TBST). After washing the membrane $(3 \times 10 \mathrm{~min})$ with TBST, the protein-antibody recognition was detected using an enhanced chemiluminescence ECL western blotting detection kit (Pierce). The emitted chemiluminescence was captured by Hyperfilm ECL film in the dark; the film was developed on a Kodak M35A X-OMAT processor (Kodak Canada, Inc.).

\section{Isolation of ovarian ZP and purification of $m Z P 1, m Z P 2$, and $\mathrm{mZP3}$}

Large numbers of ovarian ZP $\left(5-20 \times 10^{3}\right)$ were isolated by Percoll gradient ultracentrifugation of mouse ovarian homogenates, according to the previously described method (Litscher \& Wassarman 1999) with modifications. Briefly, a homogenate in triethanolamine buffer containing $1 \%$ deoxycholate was prepared from ovaries dissected from 20 to 30 mice (6-8 weeks old) and subjected to Percoll gradient ultracentrifugation. ZP (both intact and fragmented), 
sedimented as a band on the upper third of the tube at $1.02 \mathrm{~g} /$ $\mathrm{ml}$ density, were aspirated through a needle into the syringe and re-subjected to Percoll gradient ultracentrifugation. ZP collected from the second ultracentrifugation round were then washed free of Percoll solution in $25 \mathrm{mM}$ triethanolamine, $\mathrm{pH}$ $8.5,150 \mathrm{mM} \mathrm{NaCl}$ in a $15 \mathrm{ml}$ conical polypropylene tube $(1500 \mathrm{~g}, 10 \mathrm{~min}, \mathrm{RT})$. The ZP pellet was transferred into a microcentrifuge tube and was washed with PBS $(10000 \mathrm{~g}$, 5 min, RT). The final ZP pellet was stored in $50 \%$ glycerol in PBS at $-20{ }^{\circ} \mathrm{C}$. Solubilization of the isolated ZP was done by first washing the $\mathrm{ZP}$ in $\mathrm{PBS}, \mathrm{pH}$ 6.8, followed by resuspension and vortexing in $100 \mu \mathrm{l}$ of $0.2 \mathrm{M}$ sodium phosphate buffer, $\mathrm{pH}$ 6.8, containing $0.1 \%$ SDS. The ZP became solubilized by heating at $65{ }^{\circ} \mathrm{C}$ for $10 \mathrm{~min}$. After the tube was cooled down to $\mathrm{RT}$, the insoluble material was pelleted by centrifugation (14000 $\mathrm{g}$, $1 \mathrm{~min}$ ). Total amount of zona glycoproteins in the supernatant was determined by A280 using a weighted average of ZP1, ZP2, and ZP3 extinction coefficients and a 1:5:5 molar ratio for the three ZP glycoproteins in each ZP (Kerr et al. 2002). The supernatant, containing solubilized ZP, was used to purify individual ZP glycoproteins, employing two separate methods - HPLC and electroelution.

The HPLC method was as described by Litscher \& Wassarman (1999). Solubilized ZP was fractionated on a size exclusion column BioSil SEC-250 (Bio-Rad) at RT and eluted with HPLC buffer (same as the ZP solubilization buffer) at a flow rate of $0.1 \mathrm{ml} / \mathrm{min}$ with online $\mathrm{A} 280$ monitoring. Eluted fractions, $100 \mu \mathrm{l}$ each, were collected and measured for A280. The fractions of each protein peak were pooled, determined for protein amount by A280 using the calculated extinction coefficients for mZP1, mZP2, and mZP3 of 60980 , 74230 , and $35010 \mathrm{~cm}^{-1} \mathrm{M}^{-1}$ respectively (Kerr et al. 2002). From the mole amounts obtained from this calculation, the weight amounts were derived using molecular weight of 100000,120000 , and 83000 for mZP1, mZP2, and mZP3 respectively. The $\mathrm{mZP}$ fractions were then dialyzed for $24-48 \mathrm{~h}$ against distilled water at $4{ }^{\circ} \mathrm{C}$ using a tubing with a molecular mass cutoff of $30 \mathrm{kDa}$. Finally, the samples were lyophilized and stored at $-80{ }^{\circ} \mathrm{C}$ prior to use.

In the electroelution method, the solubilized ZP was first resolved by SDS-PAGE as described above. The sample was applied on several lanes, with each lane containing no more than $25 \mu \mathrm{g}$ of total ZP glycoproteins. To locate the band position of each mZP glycoprotein, $1 \mu \mathrm{g}$ of solubilized ZP was applied to a separate lane on the same gel. Following gel electrophoresis, the lane with $1 \mu \mathrm{g}$ of solubilized ZP was cut off the gel and stained with silver nitrate using the Bio-Rad Silver Stain kit to reveal the positions of mZP1 (200 kDa), mZP2 (120 kDa), and mZP3 (83 kDa). Corresponding regions of each ZP glycoprotein from lanes loaded with $25 \mu \mathrm{g}$ of solubilized ZP were marked by matching with the stained gel. The marked areas were cut to produce gel slices, which were subjected to electroelution using a Centrilutor Micro-Electroeluter (Millipore). The electroelution was carried out at a constant voltage (200 V) for $2 \mathrm{~h}$ at RT using degassed SDS-electrophoresis buffer minus SDS. The eluted proteins were washed extensively with PBS, pH 7.4, through six cycles of volume reductionresuspension (more than tenfold volume reduction each time) in Centricon YM-30 (Millipore). Solubilized ZP and each purified ZP glycoprotein were quantified by A280.

The purity of the purified ZP glycoproteins (by either HPLC or electroelution) was examined by silver staining and for $\mathrm{mZP} 2$ and $\mathrm{mZP} 3$, and was further validated by immunoblotting using a $\mathrm{mAb}$ against $\mathrm{mZP} 2$ and $\mathrm{mZP} 3$ respectively. The biological activity of total solubilized ZP and the purified ZP3 as a sperm acrosome reaction inducer was also assessed. Briefly, capacitated PGC sperm were incubated $\left(30 \mathrm{~min}, 37^{\circ} \mathrm{C}, 5 \%\right.$ $\mathrm{CO}_{2}$ ) with six ZP equivalent amounts of solubilized ZP and each individual ZP glycoproteins per microliter of the sperm suspension. The acrosome status of the sperm was determined by Coomassie blue staining (Bleil \& Wassarman 1990). Capacitated sperm untreated or treated with ovalbumin or fetuin, which were also subjected to the same electroelution as the ZP glycoproteins, served as negative controls (Carmona et al. 2002 b), whereas sperm treated with 10 M A23187 were a positive control.

\section{Binding of ASA to mZP glycoproteins by dot blotting}

Various concentrations of solubilized mouse ZP (containing the three $\mathrm{mZP}$ glycoproteins) and individual mZP glycoproteins were separately spotted to the size of $0.5 \mathrm{~cm}$ in diameter onto nitrocellulose membrane and air dried. In certain experiments, ovalbumin and fetuin, $1 \mu \mathrm{g}$ each, were also spotted on the same membrane. The membrane was blocked in 5\% nonfat milk in TBST ( $1 \mathrm{~h}, \mathrm{RT})$ and incubated ( $1 \mathrm{~h}, \mathrm{RT})$ with purified ASA $(3 \mu \mathrm{g})$ in $1 \mathrm{ml}$ of TBST. The membrane was then washed three times ( 5 min each) with TBST and incubated ( $1 \mathrm{~h}, \mathrm{RT})$ with anti-ASA antiserum (1:1000 dilution in 5\% nonfat milk). After washing three times in TBST, the membrane was incubated (1 h, RT) with HRP-conjugated goat anti-rabbit IgG (1:5000 dilution in TBST containing 5\% skim milk), and processed for chemiluminescence detection as described in the Immunoblotting section and the scanned image (in TIFF format) was subjected to densitometric analysis.

To demonstrate that the binding of ASA to mZP glycoproteins immobilized onto the nitrocellulose membrane was specific, ASA was preincubated (30 min, RT) in solution with $10 \mu \mathrm{g}$ of solubilized total $\mathrm{mZP}$ glycoprotein $(\sim 10 \times$ mole ratio to the dotted mZP glycoproteins and mZP3 on the membrane, and $\sim 5 \times$ mole ratio to the dotted $\mathrm{mZP} 2$ ) prior to co-incubation with the immobilized mZP. In alternate experiments, dextran sulfate and dextran ( $1 \mu \mathrm{M}$ each) (both from Sigma and with the size of $500 \mathrm{kDa}$ ) were included during the co-incubation of ASA with the immobilized mZP glycoproteins. Detection of ASA-mZP glycoprotein interaction was then performed as described above.

\section{Supplementary data}

This is linked to the online version of the paper at http://dx.doi. org/10.1530/REP-11-0338.

\section{Declaration of interest}

The authors declare that there is no conflict of interest that could be perceived as prejudicing the impartiality of the research reported. 


\section{Funding}

This work was funded by CIHR MOP 84420 (to $N$ Tanphaichitr), International Collaborative Research Funding from the University of Ottawa and Gothenburg University (to N Tanphaichitr), an Ontario Graduate Scholarship (to $\mathrm{H} \mathrm{Xu}$ ), and the Development and Promotion of Science and Technology Talented Project (DPST) scholarship, Thailand (to $\mathrm{N}$ Srakaew). Biognos AB, Gothenburg, is acknowledged for supporting C Koppisetty and P-G Nyholm in the docking part of this project.

\section{Acknowledgements}

We thank Ms Kessiri Kongmanas for her technical help, and together with Ms Terri van Gulik for the help in manuscript preparation.

\section{References}

Ahn VE, Faull KF, Whitelegge JP, Fluharty AL \& Prive GG 2003 Crystal structure of saposin B reveals a dimeric shell for lipid binding. PNAS 100 38-43. (doi:10.1073/pnas.0136947100)

Bleil JD \& Wassarman PM 1990 Identification of a ZP3-binding protein on acrosome intact mouse sperm by photoaffinity crosslinking. PNAS 87 5563-5567. (doi:10.1073/pnas.87.14.5563)

Bleil JD, Greve JM \& Wassarman PM 1988 Identification of a secondary sperm receptor in the mouse egg zona pellucida: role in maintenance of binding of acrosome-reacted sperm to eggs. Developmental Biology 128 376-385. (doi:10.1016/0012-1606(88)90299-0)

Brandon CI, Srivastava PN, Heusner GL \& Fayrer-Hosken RA 1997 Extraction and quantification of acrosin, $\beta$ - $N$-acetylglucosaminidase, and arylsulfatase-A from equine ejaculated spermatozoa. Journal of Experimental Zoology 279 301-308. (doi:10.1002/(SICI)1097-010X (19971015)279:3 < 301::AID-JEZ11>3.0.CO;2-C)

Buffone MG, Foster JA \& Gerton GL 2008a The role of the acrosomal matrix in fertilization. International Journal of Developmental Biology $\mathbf{5 2}$ 511-522. (doi:10.1387/ijdb.072532mb)

Buffone MG, Zhuang T, Ord TS, Hui L, Moss SB \& Gerton GL $2008 b$ Recombinant mouse sperm ZP3-binding protein (ZP3R/sp56) forms a high order oligomer that binds eggs and inhibits mouse fertilization in vitro. Journal of Biological Chemistry 283 12438-12445. (doi:10.1074/jbc.M706421200)

Burkart AD, Xiong B, Baibakov B, Jimenez-Movilla M \& Dean J 2012 Ovastacin, a cortical granule protease, cleaves ZP2 in the zona pellucida to prevent polyspermy. Journal of Cell Biology 197 37-44. (doi:10.1083/ jcb.201112094)

Carmona E, Weerachatyanukul W, Soboloff T, Fluharty AL, White D, Promdee L, Ekker M, Berger T, Buhr M \& Tanphaichitr N 2002a Arylsulfatase $A$ is present on the pig sperm surface and is involved in sperm-zona pellucida binding. Developmental Biology 247 182-196. (doi:10.1006/dbio.2002.0690)

Carmona E, Weerachatyanukul W, Xu H, Fluharty AL, Anupriwan A, Shoushtarian A, Chakrabandhu K \& Tanphaichitr N 2002b Binding of arylsulfatase $\mathrm{A}$ to mouse sperm inhibits gamete interaction and induces the acrosome reaction. Biology of Reproduction 66 1820-1827. (doi:10.1095/biolreprod66.6.1820)

Dell A, Chalabi S, Easton RL, Haslam SM, Sutton-Smith M, Patankar MS, Lattanzio F, Panico M, Morris HR \& Clark GF 2003 Murine and human zona pellucida 3 derived from mouse eggs express identical O-glycans. PNAS 100 15631-15636. (doi:10.1073/pnas.2635507100)

Dudkiewicz AB 1984 Purification of boar acrosomal arylsulfatase A and possible role in the penetration of cumulus cells. Biology of Reproduction 30 1005-1014. (doi:10.1095/biolreprod30.4.1005)
East IJ, Mattison DR \& Dean J 1984 Monoclonal antibodies to the major protein of the murine zona pellucida: effects on fertilization and early development. Developmental Biology 104 49-56. (doi:10.1016/00121606(84)90035-6)

East IJ, Gulyas BJ \& Dean J 1985 Monoclonal antibodies to the murine zona pellucida protein with sperm receptor activity: effects on fertilization and early development. Developmental Biology 109 268-273. (doi:10.1016/ 0012-1606(85)90454-3)

El-Mestrah M, Castle PI, Borossa G \& Kan FW 2002 Subcellular distribution of ZP1, ZP2, and ZP3 glycoproteins during folliculogenesis and demonstration of their topographical disposition within the zona matrix of mouse ovarian oocytes. Biology of Reproduction 66 866-876. (doi:10. 1095/biolreprod66.4.866)

Erickson RP \& Martin SR 1974 The relationship of mouse spermatozoal to mouse testicular cathepsins. Archives of Biochemistry and Biophysics 165 114-120. (doi:10.1016/0003-9861(74)90147-7)

Florman HM \& Ducibella T 2006 Fertilization in mammals. In Knobil and Neil's Physiology of Reproduction, vol 1, pp 55-112. Ed. JD Neill. New York: Elsevier.

Gaboriau D, Howes EA, Clark J \& Jones R 2007 Binding of sperm proacrosin/beta-acrosin to zona pellucida glycoproteins is sulfate and stereodependent. Synthesis of a novel fertilization inhibitor. Developmental Biology 306 646-657. (doi:10.1016/j.ydbio.2007.04.001)

Gahlay G, Gauthier L, Baibakov B, Epifano O \& Dean J 2010 Gamete recognition in mice depends on the cleavage status of an egg's zona pellucida protein. Science 329 216-219. (doi:10.1126/science. 1188178)

Green DP 1997 Three-dimensional structure of the zona pellucida. Reviews of Reproduction 2 147-156. (doi:10.1530/ror.0.0020147)

Greve JM \& Wassarman PM 1985 Sulfatases: structure, mechanism, biological activity, inhibition, and synthetic utility. Journal of Molecular Biology 181 253-264. (doi:10.1016/0022-2836(85)90089-0)

Hanson SR, Best MD \& Wong CH 2004 Sulfatases: structure, mechanism, biological activity, inhibition, and synthetic utility. Angewandte Chemie International Edition in English 43 5736-5763. (doi:10.1002/anie. 200300632)

Hiraiwa M, Martin BM, Kishimoto Y, Conner GE, Tsuji S \& O'Brien JS 1997 Lysosomal proteolysis of prosaposin, the precursor of saposins (sphingolipid activator proteins): its mechanism and inhibition by ganglioside. Archives of Biochemistry and Biophysics 341 17-24. (doi:10.1006/abbi. 1997.9958)

Hirano T, Takasaki S, Hedrick JL, Wardrip NJ, Amano J \& Kobata A 1993 O-linked neutral sugar chains of porcine zona pellucida glycoproteins. European Journal of Biochemistry 214 763-769. (doi:10.1111/j.14321033.1993.tb17978.x)

Hokke CH, Damm JBL, Penninkhof B, Aitken RJ, Kamerling JP \& Vliegenthart JFG 1994 Structure of the O-linked carbohydrate chains of porcine zona pellucida glycoproteins. European Journal of Biochemistry 221 491-512. (doi:10.1111/j.1432-1033.1994.tb18762.x)

Howes L \& Jones R 2002 Interactions between zona pellucida glycoproteins and sperm proacrosin/acrosin during fertilization. Journal of Reproductive Immunology 53 181-192. (doi:10.1016/S0165-0378(01)00101-2)

Howes E, Pascall JC, Engel W \& Jones R 2001 Interactions between mouse ZP2 glycoprotein and proacrosin; a mechanism for secondary binding of sperm to the zona pellucida during fertilization. Journal of Cell Science 114 4127-4136.

lamsaard S, Vanichviriyakit R, Hommalai G, Saewu A, Srakaew N, Withyachumnarnkul B, Basak A \& Tanphaichitr N 2011 Enzymatic activity of sperm proprotein convertase is important for mammalian fertilization. Journal of Cellular Physiology 226 2817-2826. (doi:10.1002/jcp.22626)

Inoue N, Ikawa M, Isotani A \& Okabe M 2005 The immunoglobulin superfamily protein Izumo is required for sperm to fuse with eggs. Nature 434 234-238. (doi:10.1038/nature03362)

Jimenez-Movilla M \& Dean J 2011 ZP2 and ZP3 cytoplasmic tails prevent premature interactions and ensure incorporation into the zona pellucida. Journal of Cell Science 124 940-950. (doi:10.1242/jcs.079988)

Kawai Y, Hama T, Mayumi T, Okabe M, Matzno S, Kohama Y \& Mimura T 1989 Flow cytometric analysis of mouse sperm using monoclonal antisperm antibody OBF13. Journal of Reproductive Immunology 16 71-82. (doi:10.1016/0165-0378(89)90007-7) 
Kerr CL, Hanna WF, Shaper JH \& Wright WW 2002 Characterization of zona pellucida glycoprotein 3 (ZP3) and ZP2 binding sites on acrosome-intact mouse sperm. Biology of Reproduction 66 1585-1595. (doi:10.1095/biolreprod66.6.1585)

Kim KS \& Gerton GL 2003 Differential release of soluble and matrix components: evidence for intermediate states of secretion during spontaneous acrosomal exocytosis in mouse sperm. Developmental Biology 264 141-152. (doi:10.1016/j.ydbio.2003.08.006)

Laemmli UK 1970 Cleavage of structural proteins during the assembly of the head of bacteriophage T4. Nature 227 680-685. (doi:10.1038/ 227680a0)

Lin YN, Roy A, Yan W, Burns KH \& Matzuk MM 2007 Loss of zona pellucida binding proteins in the acrosomal matrix disrupts acrosome biogenesis and sperm morphogenesis. Molecular and Cellular Biology 27 6794-6805. (doi:10.1128/MCB.01029-07)

Litscher ES \& Wassarman PM 1999 Purification and functional analysis of mouse egg zona pellucida glycoproteins. In Oocytes and Embryos, A Comparative Methods Approach, pp 10-22. Ed. JD Richter. Oxford: Oxford University Press.

Litscher ES, Williams Z \& Wassarman PM 2009 Zona pellucida glycoprotein ZP3 and fertilization in mammals. Molecular Reproduction and Development 76 933-941. (doi:10.1002/mrd.21046)

Lukatela G, Krauss N, Theis K, Selmer T, Gieselmann V, von Figura K \& Saenger W 1998 Crystal structure of human arylsulfatase A: the aldehyde function and the metal ion at the active site suggest a novel mechanism for sulfate ester hydrolysis. Biochemistry 37 3654-3664. (doi:10.1021/ bi9714924)

Lyng R \& Shur BD 2007 Sperm-egg binding requires a multiplicity of receptor-ligand interactions: new insights into the nature of gamete receptors derived from reproductive tract secretions. Society of Reproduction and Fertility Supplement 65 335-351.

Moreno RD, Bustamante E, Schatten G \& Barros C 2002 Inhibition of mouse in vitro fertilization by an antibody against a unique 18-amino acid domain in the polysulfate-binding domain of proacrosin/acrosin. Fertility and Sterility 77 812-817. (doi:10.1016/S0015-0282(01)03245-9)

Mori E, Baba T, Iwanatsu A \& Mori T 1993 Purification and characterization of a 38-kDa protein, sp38, with zona pellucida-binding property from porcine epididymal sperm. Biochemical and Biophysical Research Communications 196 196-202. (doi:10.1006/bbrc.1993.2234)

Nakanishi T, Ikawa M, Yamada S, Toshimori K \& Okabe M 2001 Alkalinization of acrosome measured by GFP as a pH indicator and its relation to sperm capacitation. Developmental Biology 237 222-231. (doi:10.1006/dbio.2001.0353)

Nikolajczyk BS \& O'Rand MG 1992 Characterization of rabbit testis $\beta$-galactosidase and arylsulfatase A: purification and localization in spermatozoa during the acrosome reaction. Biology of Reproduction $\mathbf{4 6}$ 366-378. (doi:10.1095/biolreprod46.3.366)

Noguchi S \& Nakano M 1993 Structural characterization of the N-linked carbohydrate chains from mouse zona pellucida glycoproteins ZP2 and ZP3. Biochimica et Biophysica Acta 1158 217-226. (doi:10.1016/03044165(93)90018-4)

Oehninger S, Clark GF, Acosta AA \& Hodgen GD 1991 Nature of the inhibitory effect of complex saccharide moieties on the tight binding of human spermatozoa to the human zona pellucida. Fertility and Sterility 55 165-169.

Pang PC, Chiu PC, Lee CL, Chang LY, Panico M, Morris HR, Haslam SM, Khoo KH, Clark GF, Yeung WS et al. 2011 Human sperm binding is mediated by sialyl-Lewis ( $\mathrm{x}$ ) on the zona pellucida. Science $\mathbf{3 3 3}$ 1761-1764. (doi:10.1126/science.1207438)

Rankin TL, Coleman JS, Epifano O, Hoodbhoy T, Turner SG, Castle PE, Lee E, Gore-Langton R \& Dean J 2003 Fertility and taxon-specific sperm binding persist after replacement of mouse sperm receptors with human homologs. Developmental Cell 5 33-43. (doi:10.1016/S1534-5807 (03)00195-3)

Rattanachaiyanont M, Weerachatyanukul W, Leveille MC, Taylor T, D'Amours D, Rivers D, Leader A \& Tanphaichitr N 2001 Anti-SLIP1reactive proteins exist on human sperm and are involved in zona pellucida binding. Molecular Human Reproduction 7 633-640. (doi:10.1093/molehr/7.7.633)

Schenk M, Koppisetty CA, Santos DC, Carmona E, Bhatia S, Nyholm PG \& Tanphaichitr N 2009 Interaction of arylsulfatase-A (ASA) with its natural sulfoglycolipid substrates: a computational and site-directed mutagenesis study. Glycoconjugate Journal 26 1029-1045. (doi:10.1007/s10719008-9222-9)

Shimizu S, Tsiko M \& Dean J 1983 In vitro biosynthesis of three sulfated glycoproteins of murin zonae pellucidae by oocytes grown in follicle culture. Journal of Biological Chemistry 258 5858-5863.

Takasaki S \& Kobata A 1986 Asparagine-linked sugar chains of fetuin: occurrence of tetrasialyl triantennary sugar chains containing the gal $\beta(1-3)$ GlcNAc sequence. Biochemistry 25 5709-5715. (doi:10.1021/ bi00367a054)

Takasaki S, Mori E \& Mori T 1999 Structures of sugar chains included in mammalian zona pellucida glycoproteins and their potential roles in sperm-egg interaction. Biochimica et Biophysica Acta 1473 206-215. (doi:10.1016/S0304-4165(99)00180-4)

Tanphaichitr N, Bou Khalil M, Weerachatyanukul W, Kates M, Xu H, Carmona E, Attar M \& Carrier D 2003 Physiological and biophysical properties of male germ cell sulfogalactosylglycerolipid. In Lipid Metabolism and Male Fertility, vol 11, pp 125-148. Ed. S De Vriese. Champaign, IL: AOCS Press.

Tanphaichitr N, Carmona E, Bou Khalil M, Xu H, Berger T \& Gerton GL 2007 New insights into sperm-zona pellucida interaction: involvement of sperm lipid rafts. Frontiers in Bioscience 12 1748-1766. (doi:10.2741/2186)

Tantibhedhyangkul J, Weerachatyanukul W, Carmona E, Xu $H_{\text {, }}$ Anupriwan A, Michaud D \& Tanphaichitr N 2002 Role of sperm surface arylsulfatase $\mathrm{A}$ in mouse sperm-zona pellucida binding. Biology of Reproduction 67 212-219. (doi:10.1095/biolreprod67.1.212)

Topfer-Petersen E, Ekhlasi-Hundrieser M \& Tsolova M 2008 Glycobiology of fertilization in the pig. International Journal of Developmental Biology 52 717-736. (doi:10.1387/ijdb.072536et)

Towbin H \& Gordon J 1984 Immunoblotting and dot immunobindingcurrent status and outlook. Journal of Immunological Methods 72 313-340. (doi:10.1016/0022-1759(84)90001-2)

Waheed A \& Van Etten RL 1980 Chemical characterization and substrate specificity of rabbit liver aryl sulfatase A. Biochimica et Biophysica Acta 614 92-101.

Wassarman PM \& Litscher ES 2008 Mammalian fertilization: the egg's multifunctional zona pellucida. International Journal of Developmental Biology 52 665-676. (doi:10.1387/ijdb.072524pw)

Weerachatyanukul W, Xu H, Anupriwan A, Carmona E, Wade M, Hermo L, da Silva SM, Rippstein P, Sobhon P, Sretarugsa P et al. 2003 Acquisition of arylsulfatase $A$ onto the mouse sperm surface during epididymal transit. Biology of Reproduction 69 1183-1192. (doi:10.1095/biolreprod. 102.010231)

White D, Weerachatyanukul W, Gadella B, Kamolvarin N, Attar M \& Tanphaichitr N 2000 Role of sperm sulfogalactosylglycerolipid in mouse sperm-zona pellucida binding. Biology of Reproduction 63 147-155. (doi:10.1095/biolreprod63.1.147)

Wu A, Anupriwan A, lamsaard S, Chakrabandhu K, Santos DC, Rupar T, Tsang BK, Carmona E \& Tanphaichitr N 2007 Sperm surface arylsulfatase A can disperse the cumulus matrix of cumulus oocyte complexes. Journal of Cellular Physiology 213 201-211. (doi:10.1002/jcp.21113)

Xu H, Kongmanas K, Kadunganattil S, Smith CE, Rupar T, Goto-Inoue N, Hermo L, Faull KF \& Tanphaichitr N 2011 Arylsulfatase A deficiency causes seminolipid accumulation and lysosomal disorder in Sertoli cells. Journal of Lipid Research 52 2187-2197. (doi:10.1194/jlr.M019661)

Yonezawa N, Aoki H, Hatanaka Y \& Nakano M 1995 Involvement of $\mathrm{N}$-linked carbohydrate chaines of pig zona pellucida in sperm-egg binding. European Journal of Biochemistry 233 35-41. (doi:10.1111/j. 1432-1033.1995.035_1.x)

Yu Y, Vanhorne J \& Oko R 2009 The origin and assembly of a zona pellucida binding protein, IAM38, during spermiogenesis. Microscopic Research and Techniques 72 558-565. (doi:10.1002/jemt.20696)

Received 10 September 2011

First decision 4 November 2011

Revised manuscript received 5 June 2012

Accepted 8 June 2012 